\title{
TLR9- and Src-dependent expression of Krueppel-like factor 4 controls interleukin-10 expression in pneumonia
}

\author{
Janine Zahlten*, Robert Steinicke*, Wilhelm Bertrams*, Andreas C. Hocke*, \\ Stefanie Scharf*, Bernd Schmeck*, Martin Witzenrath*, Sven Hammerschmidt ${ }^{\star}$, \\ Norbert Suttorp* and Stefan Hippenstiel*
}

ABSTRACT: The release of potent pro-inflammatory mediators is crucial to mounting an efficient host response during infection. However, excessive inflammation may lead to deleterious tissue damage. This is highlighted in severe pneumococcal pneumonia, in which the delicate balance between a robust inflammatory response necessary to kill pneumococci and the loss of organ function determines the outcome of the disease.

We assessed the regulation of the potent anti-inflammatory cytokine interleukin (IL)-10 in pneumococcal infection via Western blot, ELISA and chromatin immunoprecipitation analysis.

Streptococcus pneumoniae induced IL-10 expression in mouse lungs and human lung epithelial cells. Pneumococcal infection resulted in a strong induction of Krueppel-like factor (KLF)4 expression in vivo and in vitro. The induction of both IL-10 and KLF4 is mediated by a pathway involving bacterial DNA, Toll-like receptor (TLR)9, MyD88 and Src kinase. KLF4 is recruited to the il10 promoter, and small-interfering RNA-mediated knockdown of KLF4 expression blocked IL-10 expression during pneumococcal infection.

In conclusion, KLF4 is induced in a bacterial DNA-TLR9-Src-dependent manner and regulates IL-10 expression, linking the detection of bacterial DNA by TLR9 to the control of an inflammatory response.

KEYWORDS: Infection, inflammation, innate immunity, signal transduction, Streptococcus pneumoniae

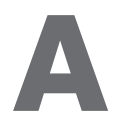

rapid, robust and efficient innate immune response defends mammals against invading pathogens. In combination with, for example, pathogen-derived toxins, the pro-inflammatory and toxic agents released from human cells impair tissue function. Thus, the innate immune response must be sufficient to kill the pathogens, but it also needs to be tightly controlled to ensure minimal tissue damage and host survival. This delicate balance is highlighted in pneumonia, which represents the third leading cause of death worldwide [1]. On the one hand, local elimination of bacteria by the host response prevents the distribution of infection and inflammation throughout the lung and body during pneumonia [2]. On the other hand, efficient oxygenation of the host must be guaranteed on, literally, a minute-tominute basis, despite the ongoing combat between the host and the pathogen.
Most cases of community-acquired pneumonia are due to infection with Streptococcus pneumoniae (pneumococci) [3]. The activation of Toll-like receptor (TLR)2, TLR4 and TLR9 seems to contribute to the activation of the innate immune response by pneumococci [4-6]. These receptors initiate a strong release of cytokines, resulting in the characteristic infiltration of the lung by polymorphonuclear leukocytes [2]. Despite the vital need for a balanced inflammatory reaction in pneumonia, little is known about how inflammation in pneumonia is controlled.

Krueppel-like transcription factors (KLFs) form a subclass of 21 zinc finger-containing transcription factors expressed in mammals [7]. KLF4 was first identified as an important factor for the establishment of the skin barrier [8]. Recent studies suggest that KLF4 is involved in the regulation of cytokine production in leukocytes $[9,10]$.

\section{AFFILIATIONS}

*Dept of Internal Medicine/Infectious Diseases and Respiratory Medicine, Charité - Universitätsmedizin Berlin, Berlin,

\#Dept of Molecular Pulmonology,

German Center for Lung Research,

Universities of Giessen and Marburg Lung Center, Philipps University

Marburg, Marburg, and

"Dept of Genetics of

Microorganisms, Interfaculty Institute

of Genetics and Functional

Genomics, Ernst-Moritz-Arndt-

University, Greifswald, Germany.

CORRESPONDENCE

S. Hippenstiel

Dept of Internal Medicine/Infectious

Diseases and Respiratory Medicine

Charité - Universitätsmedizin Berlin

Augustenburger Platz 1

13353 Berlin

Germany

E-mail: Stefan.hippenstiel@

charite.de

Received:

Nov 112011

Accepted after revision:

April 022012

First published online:

May 312012 
By using S. pneumoniae infection as a model, we analysed the regulation of anti-inflammatory interleukin (IL)-10 expression. We report that KLF4 is induced in a bacterial DNA-TLR9-Srcdependent manner and regulates IL-10 expression, linking the detection of bacterial DNA by TLR9 to the control of an antiinflammatory response.

\section{MATERIAL AND METHODS Materials}

The src kinase inhibitor, PP2, and its control, PP3, were purchased from Calbiochem (Merck, Darmstadt, Germany). ODN M362 and ODN TTAGGG were purchased from InvivoGen (San Diego, CA, USA). All other chemicals used were of analytical grade and obtained from commercial sources.

\section{Bacterial strains and bacterial products}

Pneumonia was induced in mice by infection with $S$. pneumoniae serotype 3 strain PN36 (NCTC 7978). S. pneumoniae R6x and R6x ${ }^{\Delta p l y}$ (pneumolysin (Ply)-deficient) strains used for the in vitro studies are unencapsulated derivatives of serotype 2 strain D39. Pneumococci were plated on blood agar plates overnight then cultured in Todd Hewitt broth supplemented with 5\% yeast extract to midlog phase (optical density at $600 \mathrm{~nm}$ (OD600) 0.3), harvested by centrifugation and resuspended as recommended in cell culture medium. Heat inactivation of R6x was performed for $1 \mathrm{~h}$ at $56^{\circ} \mathrm{C}$. For the isolation of bacterial DNA, pneumococci were cultured (OD600 1.0), harvested by centrifugation, resuspended in $\mathrm{N}$-tris(hydroxymethyl)methyl-2-aminoethane sulfonic acid, lysed and treated with lysozyme, mutanolysin, RNase, pronase E and sarcosyl. DNA was extracted and precipitated with phenol, sodium acetate and 2-propanol. Ply was a kind gift from T. Mitchell (Biomedical Research Centre, University of Glasgow, Glasgow, UK).

\section{Human cell lines and DNA}

Transformed human bronchial epithelial cells (BEAS)-2B cells and human embryonic kidney (HEK)293 were purchased from the American Type Culture Collection (Rockville, MD, USA). Human primary small airway epithelial cells (SAECs) were obtained from Clonetics/Cambrex (Taufkirchen, Germany). Cells were cultured and infected as described previously [11]. Human DNA from BEAS-2B cells was prepared using the QIAampDNA Mini Kit (Qiagen, Hilden, Germany) following manufacturer's instructions.

\section{Mouse pneumonia model}

All animal procedures were performed according to the Helsinki convention for the use of animals and approved by the State Office of Health and Social Affairs in Berlin (Germany). 6-week-old female C57BL/6 mice were anaesthetised, infected and then sacrificed at the indicated time-points. Lung bacterial load reaches its maximum $60 \mathrm{~h}$, septic dissemination commences $\sim 24 \mathrm{~h}$ and mice become moribund $60-120 \mathrm{~h}$ after infection [12]. Removed lungs were frozen in liquid nitrogen and bronchoalveolar lavage (BAL) was performed.

\section{IL-10 ELISA}

IL-10 production in the supernatants or BAL was assessed by commercial ELISA (Becton Dickinson GmbH, Heidelberg, Germany). To achieve more sensitive IL-10 detection, we generated standard dilutions from $3.9 \mathrm{ng}$ to $250 \mathrm{ng}$ for the in vitro studies. For all ELISA data, cell viability was confirmed by measurement of lactate dehydrogenase in the same supernatants (data not shown).

\section{Western blotting}

Cells were stimulated as indicated, washed twice and harvested. Mouse lungs were snap-frozen in liquid nitrogen and pulverised. Cell or lung homogenates were lysed in buffer containing NP40 and subjected to Western blotting. Membranes were exposed to antibodies for IL-10 (Abcam, Cambridge, MA), KLF2, KLF4, TLR9, MyD88, extracellular signal-regulated kinase (ERK)2, actin (all Santa Cruz Biotechnology, Inc., Heidelberg, Germany), cyclooxygenase (COX)2, focal adhesion kinase (Upstate Biotechnology, Lake Placid, NY, USA) overnight at $4^{\circ} \mathrm{C}$ and subsequently incubated with secondary antibodies (IRDye 800-labelled anti-mouse or anti-goat, or Cy5.5-labeled anti-rabbit). Proteins were detected using an Odyssey infrared imaging system (LI-COR Inc., Bad Homburg, Germany).

\section{Plasmids and transfection procedures}

HEK293 cells were co-transfected using the calcium phosphate precipitation method according to the manufacturer's instructions (Clonetech, Palo Alto, CA, USA). Luciferase reporters dependent on KLF4 (Z-Y. Chen, Boston University School of Medicine, Boston, MA, USA) or IL-10 (C. Wehner, KKG Entzuendliche Lungenerkrankungen der GSF, Gauting, Germany) (0.2 $\mu \mathrm{g}$ each), or $0.1 \mu \mathrm{g}$ pRL-TK (Promega GmbH, Mannheim, Germany) plasmid were used as indicated. BEAS2B cells were transfected using Fugene6 (Roche Applied Science, Mannheim, Germany). Firefly and Renilla luciferase activity was measured by using a Dual-Luciferase Reporter Assay System (Promega GmbH, Mannheim, Germany).

\section{RNA interference}

Small interferring RNA (siRNA) lipoplexes were generated with AtuFECT01 for transfection (Silence Therapeutics AG, Berlin, Germany) using $2 \mu \mathrm{g}$ siRNA per $10^{6}$ cells [13]. siRNA sequences purchased from Ambion (Ambion Ltd, Huntington, Cambridge, UK) are shown in table S1.

\section{Chromatin immunoprecipitation}

BEAS-2B cells were stimulated as indicated, and chromatin immunoprecipitation (ChIP) was performed as described previously $[4,6,14]$ with a il10 promoter-specific primer pair (forward: tcgaggcgaccgcgacagt; reverse: ggagcagcgcgtcgctga). Immunoprecipitations were carried out with antibodies against KLF4 and RNA polymerase II (Santa Cruz Biotechnology). Equal amounts of input DNA were controlled by gel electrophoresis.

\section{Statistics}

Data are shown as mean \pm SEM for at least three independent experiments performed either in duplicate (in vitro) or five times (in vivo). One-way ANOVA was used for the analysis of the data, and the main effects were compared by Bonferroni's post-test. Figures $1 \mathrm{a}$ and $5 \mathrm{f}$ were analysed by unpaired t-test.

\section{RESULTS}

\section{Pneumococci-induced IL-10 expression}

We measured increased IL-10 protein expression in the BAL (fig. 1a) as well as in the lung tissue (fig. 1b) of pneumococciinfected mice. Furthermore, R6x pneumococci induced expression 

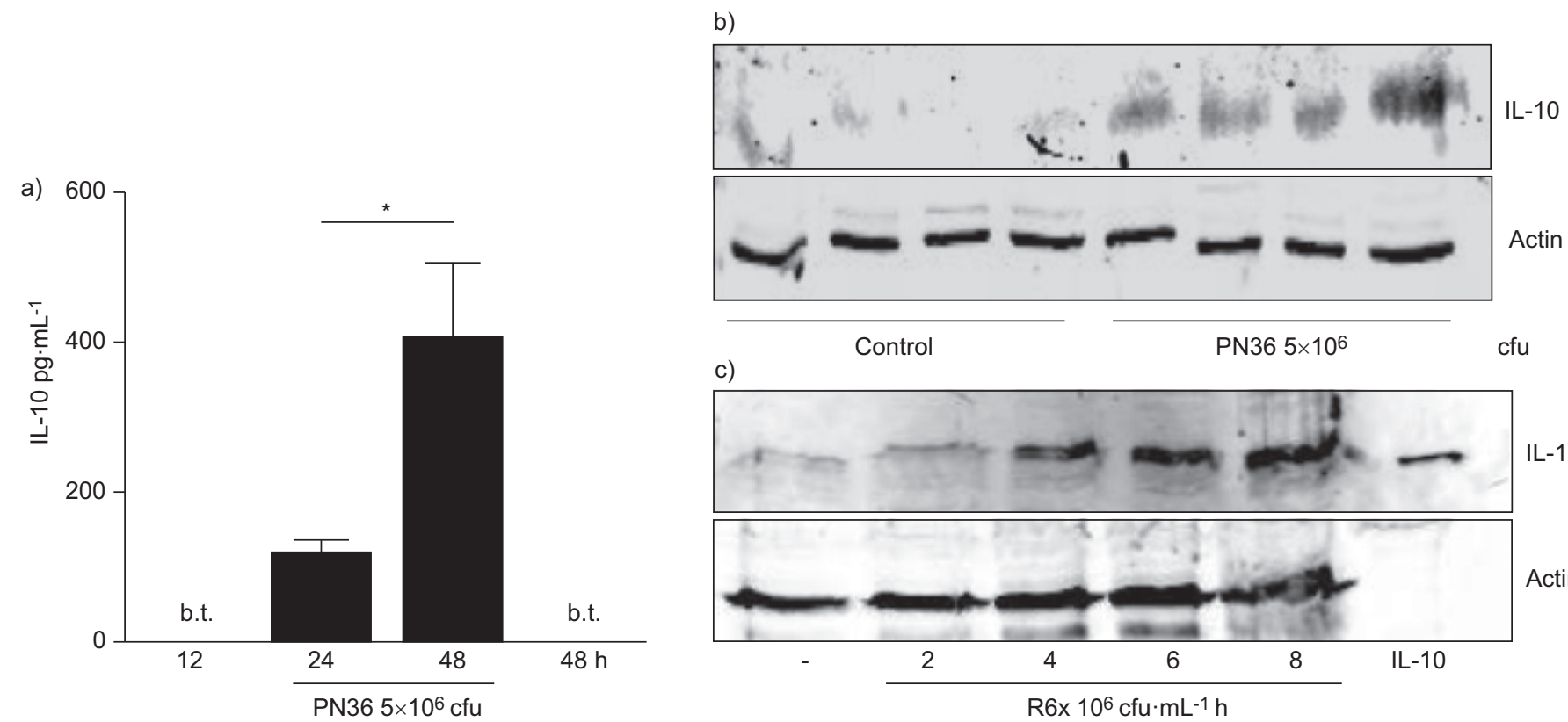

c)
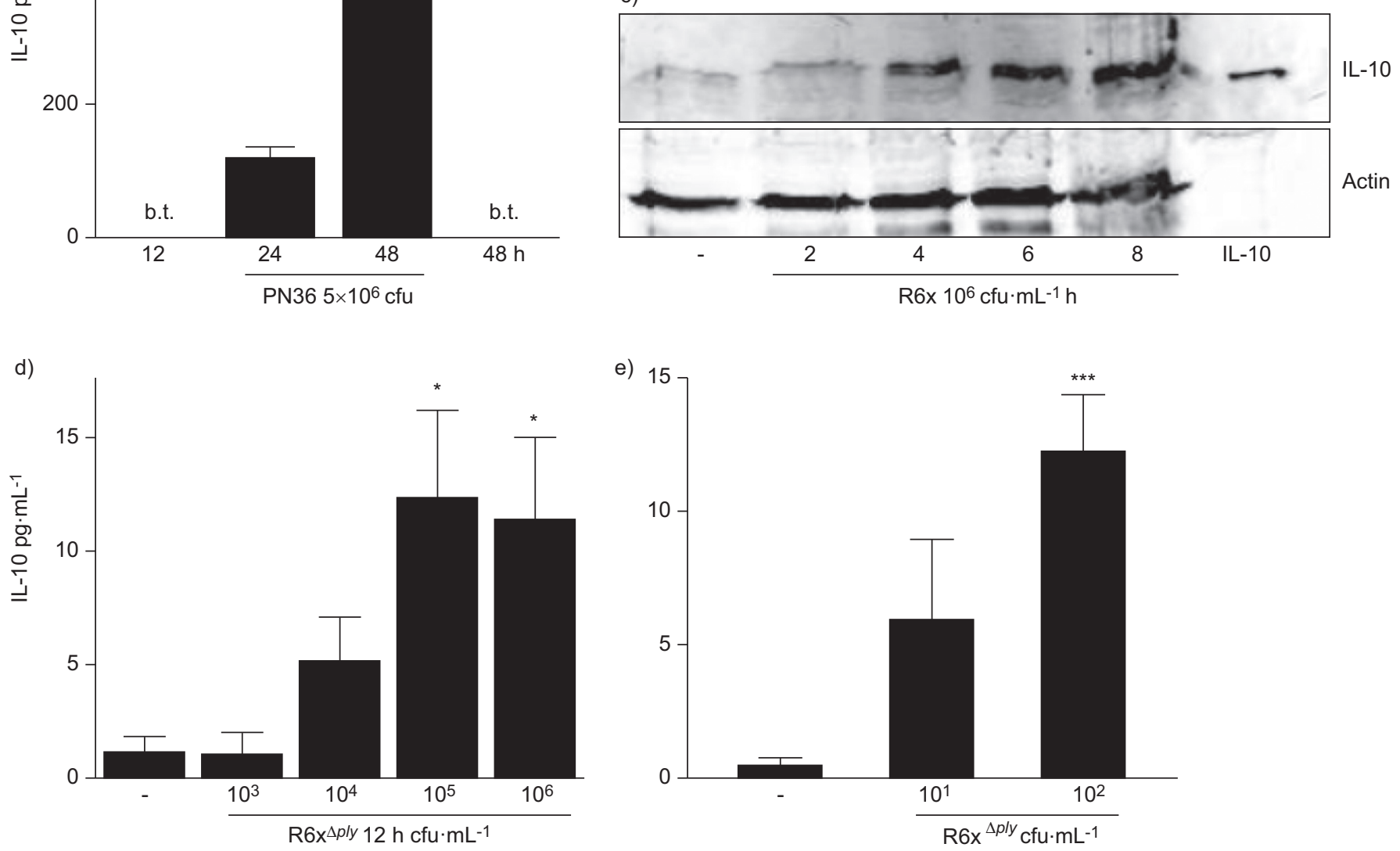

FIGURE 1. Pneumococci-induced interleukin (IL)-10 expression. Infection of mice with Streptococcus pneumoniae induced IL-10 expression in vivo in a) bronchoalveolar lavage (BAL), five mice per group and b) mouse lung tissue (four control mice or mice infected with $5 \times 10^{6}$ cfu PN36 for 48 h) as shown by a) ELISA or b) Western blot, respectively. Infection of c) bronchial epithelial cells (BEAS)-2B cells with R6x ( $10^{6} \mathrm{cfu} \cdot \mathrm{mL}^{-1}$; Western blot) or d) small airway epithelial cells (SAECs) and e) human embryonic kidney (HEK)293 with R6x ${ }^{\Delta p l y}$ for d) $12 \mathrm{~h}$ or e) $24 \mathrm{~h}$ resulted in increased IL-10 production in vitro. A total of $5 \mathrm{ng}$ of recombinant IL-10 was loaded as a positive control in b). Western blots represent one experiment out of three. Actin detection demonstrates equal protein loading. b.t.: below threshold. ${ }^{*}: p<0.05 ;{ }^{* * *}: p<0.001$.

of IL-10 in BEAS-2B cells (fig. 1c and fig. S1). Similarly, the Plydeficient mutant $\left(\mathrm{R}^{\mathrm{A}} \mathrm{x}^{\mathrm{\Delta ply}}\right)$ induced IL-10 expression in human primary SAECs (fig. 1d) and in HEK293 cells (fig. 1e).

\section{Pneumococci-induced expression of KLF4 in vitro and in vivo}

Exposure of BEAS-2B cells or SAEC to pneumococcus induced a time- (fig. 2a and b) and dose-dependent (figs S2a and b) expression of KLF4 in vitro. Moreover, increased KLF4 expression was also detected in infected mouse lungs (fig. 2c).

KLF4-reliant expression of IL-10

ChIP experiments were conducted to test the hypothesis that the IL-10 expression is regulated by KLF4 in pneumococci-infected human lung cells. Analysis of pneumococci-infected BEAS-2B cells showed a recruitment of KLF4 and RNA polymerase II to the il10 promotor (fig. 3a). Ablation of KLF4 expression by specific siRNA (fig. S3) abolished pneumococci-induced IL-10 secretion (fig. $3 b$ ).

\section{KLF4 expression in lung cells is induced by TLR9}

Abolishment of the TLR-related adaptor molecule MyD88 by siRNA reduced pneumococci-related KLF4 expression (fig. S4a and b). Ply-deficient pneumococci induced KLF4 protein expression in HEK293 cells (fig. S4c), which are known to be unresponsive to TLR4 stimulation [6]. Additionally, Ply and lipopolysaccharide (LPS) failed to induce KLF4 expression but stimulated COX2 or KLF2 expression, respectively, in 
a)

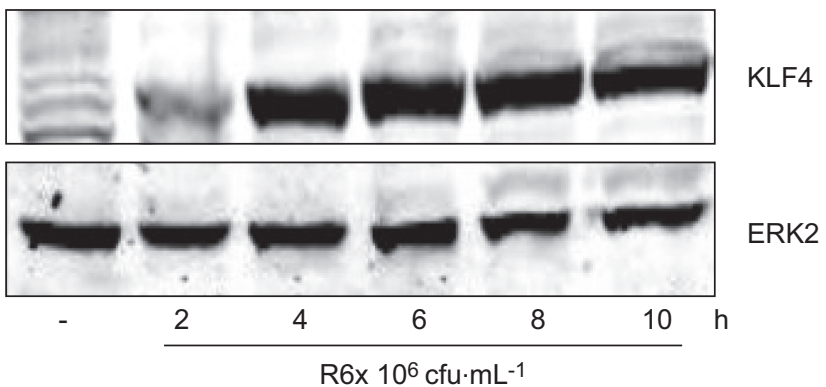

b)

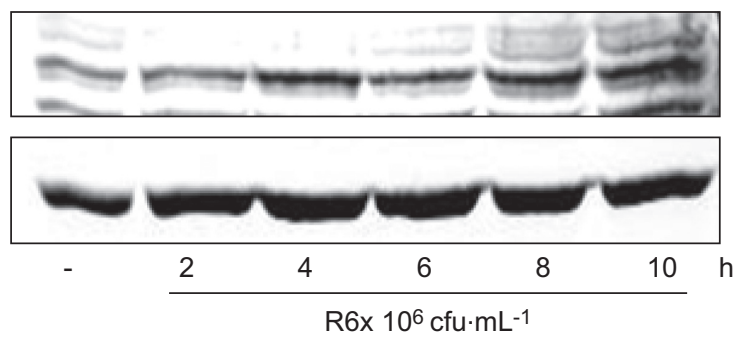

c)

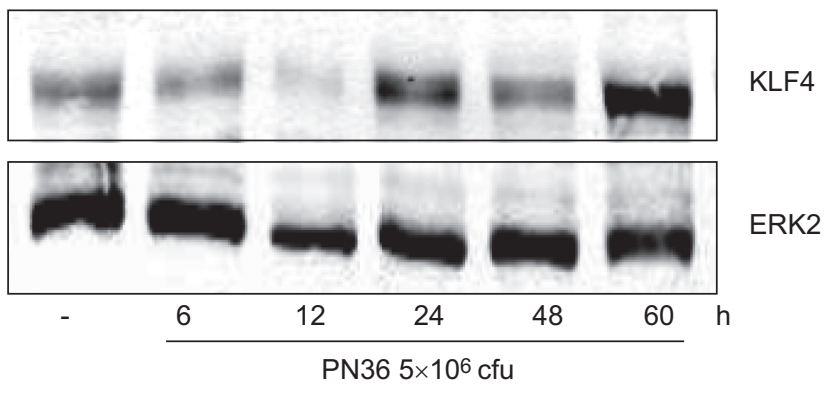

FIGURE 2. Pneumococci-induced Krueppel-like factor (KLF)4 expression. Time-dependent induction of KLF4 protein expression in a) bronchial epithelial cells (BEAS)-2B and b) small airway epithelial cells, and in c) PN36 $\left(5 \times 10^{6}\right.$ colonyforming units (cfu))-infected mouse lungs is shown. An antibody against extracellular signal-regulated kinase (ERK)2 was used as a loading control. One representative blot out of three independent experiments is shown.

BEAS-2B cells (fig. S4d and e). These results indicate that neither Ply nor TLR4 activation contributed to KLF4 expression in lung epithelial cells. Furthermore, exposure of BEAS-2B cells to heat-inactivated pneumococci failed to induce KLF4 expression while still inducing COX2 expression (fig. S4f), indicating that viable pneumococci are required to trigger KLF4 expression in lung cells. Assuming that killed pneumococci are still recognised by TLR2 [11] and that bacteriuminduced TLR9-dependent signalling is reduced by heat inactivation [5], we tested TLR9 as a possible receptor for KLF4 induction.

Both the TLR9 agonist ODN M362 (fig. 4a) and pneumococcal DNA (fig. 4b) induced expression of KLF4 in BEAS-2B cells, whereas the addition of the inhibitory CpG motif TTAGGG blocked pneumococci-dependent induction of KLF4 expression (fig. 4c). Finally, the reduction of TLR9 expression by siRNA (fig. S4g) abolished pneumococci-related KLF4 expression in BEAS-2B cells (fig. 4d). a)
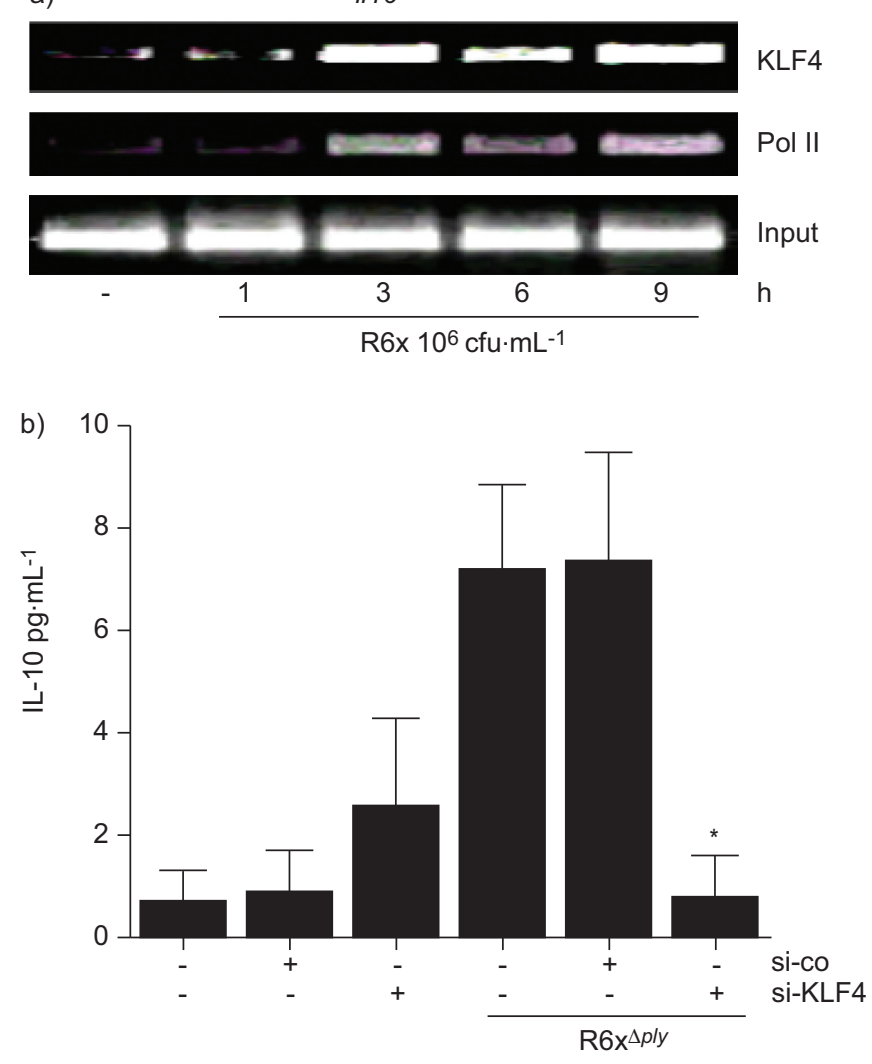

FIGURE 3. Krueppel-like factor (KLF)4-regulated interleukin (IL)-10 expression. a) A time-dependent recruitment of KLF4 and RNA polymerase II (Pol II) to the il10 promoter is shown by chromatin immunoprecipitation in bronchial epithelial cells (BEAS)-2B cells after R6x stimulation. One representative gel out of three independent experiments is shown. b) Silencing of KLF4 expression ( $96 \mathrm{~h}$ posttransfection) by specific small interfering (si)RNA (si-KLF4), but not control siRNA (si-co), reduced R6x ${ }^{\Delta D / y}\left(10^{2}\right.$ colony-forming units (cfu) $\mathrm{mL}^{-1}, 24$ h)-related IL-10 expression in human embryonic kidney (HEK)293 cells. Data are representative of three independent experiments, each performed in duplicate. *: $p<0.05$.

\section{Recognition of pneumococcal DNA-induced TLR9- dependent IL-10 expression}

Given that BEAS-2B and HEK293 cells express TLR9 (fig. S5a and b), but HEK293 cells do not express TLR2 or TLR4 [15], the exposure of these cells to the TLR9 agonist ODN M362 increased IL-10 production (fig. 5a and fig. S5c). Pneumococci-related IL10 expression in HEK293 cells was dose-dependently blocked by pre-incubation of epithelial cells with the ODN M362 antagonist TTAGGG (fig. 5b). Experiments using pneumococcal DNA showed an increase in IL-10 expression in HEK293 cells, whereas human DNA had no detectable effect (fig. 5c). Pneumococci-related IL-10 release was abolished by siRNAmediated ablation of MyD88 (fig. S5D) or TLR9 (fig. 5d).

\section{KLF4 induction and IL-10 expression are regulated by Src kinase}

1-h pre-incubation of cells with the Src inhibitor PP2 (but not with its inactive control compound PP3) blocked KLF4 expression in R6x-infected cells (fig. 6a). Accordingly, PP2 (but not PP3) inhibited IL-10 expression in pneumococciinfected HEK293 cells (fig. 6b). 
a)

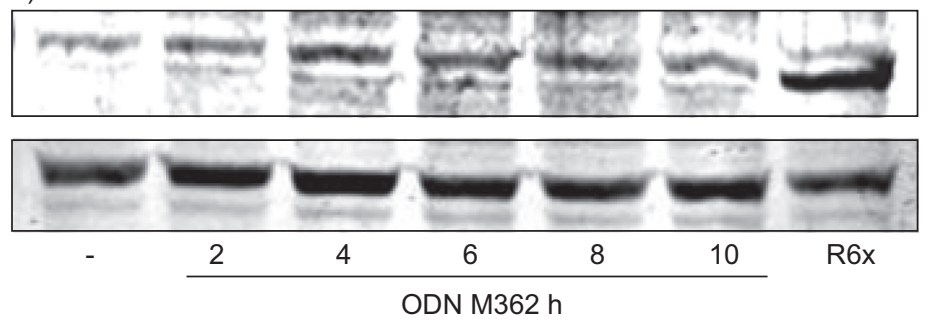

c)

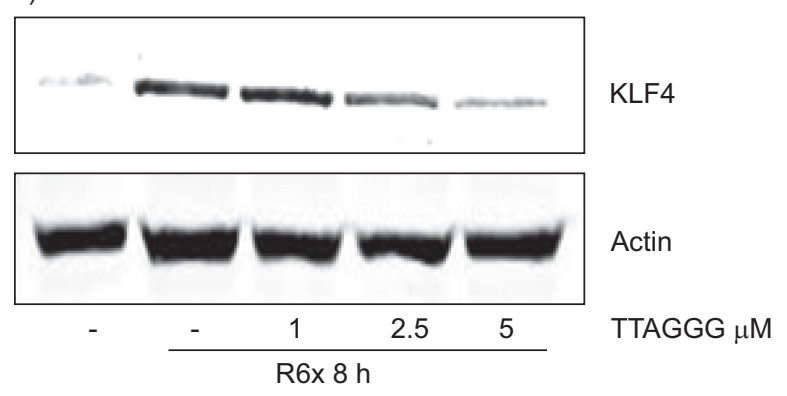

b)

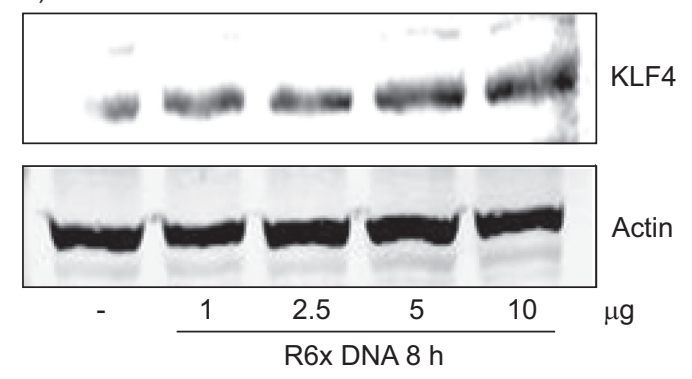

d)

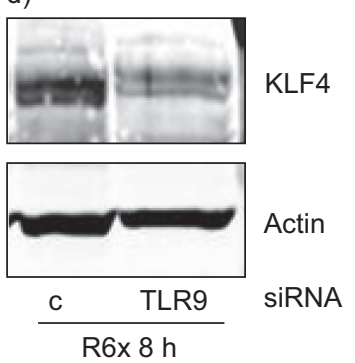

FIGURE 4. Toll-like receptor (TLR)9-dependent expression of Krueppel-like factor (KLF)4. a) Stimulation of bronchial epithelial cells (BEAS)-2B with ODN M362 $\left(1 \mu \mathrm{g} \cdot \mathrm{mL}^{-1}\right)$ and b) stimulation with different concentrations of R6x DNA (8 h)-induced KLF4 expression. c) Pre-incubation of BEAS-2B with TTAGGG reduced KLF4 protein levels following R6x stimulation for $8 \mathrm{~h}$, as did d) transfection of BEAS-2B with TLR9 siRNA for $96 \mathrm{~h}$. All Western blots are representative blots out of three independent experiments. Actin was used as a loading control. c: control.

\section{DISCUSSION}

Herein, we demonstrate bacterial DNA-dependent expression of KLF4 that subsequently induces anti-inflammatory IL-10 expression in human lung epithelial cells. Bacterial DNA or CpG-recognition by TLR9 activated Src kinase-dependent signalling, which subsequently increased KLF4 expression. Thus, activation of a TLR9-Src-KLF4-dependent pathway may be important for controlling inflammatory reactions.

The control and termination of an inflammatory reaction is important for the maintenance of tissue integrity, but much less emphasis is directed toward mechanisms controlling inflammation when compared with those initiating the innate immune response. After antibiotic treatment, it could be speculated that an ongoing strong innate immune response may be more deleterious for the host than beneficial. Furthermore, selective manipulation of anti-inflammation and resolution may allow for the development of adjuvant therapies in severe infections such as pneumonia.

Several reports indicated that pneumococci are recognised by the host via TLR2, TLR4 and TLR9. Ply is an important virulence factor of S. pneumoniae that activates host cells, at least in part, via stimulation of TLR4 [4, 6]. The LPS-TLR4 pathway was shown to induce KLF4 expression in macrophages $[9,10]$. As shown in this study, stimulation with TLR4 ligands (LPS or Ply) did not promote KLF4 expression in lung epithelium. In addition, Plydeficient pneumococci induced KLF4 expression, and this expression was also induced by these mutants in HEK293 cells, which are known to be unresponsive to TLR4 ligands [6]. This suggested that TLR4 is not involved in the pneumococcimediated KLF4 induction in human lung epithelium. Remarkably, heat-inactivated pneumococci also failed to induce
KLF4 expression. Heat inactivation of pneumococci leads to the destruction of Ply [4] and reduced sensitivity to TLR9 signalling [5], although they are still able to activate cells via TLR2 [5, 11]. Given that the stimulation of TLR2 by Pam3Cys also failed to induce KLF4 expression in embryonic stem cells [16], we excluded this receptor as a possible KLF4 inducer. In contrast, CpG-oligonucleotides mimicking bacterial DNA and pneumococcal DNA induced KLF4, which could be inhibited by the antagonising oligonucleotide TTAGGG. In line with these data, knockdown of TLR9 or MyD88, which is involved in the TLR9 signalling cascade [17], also reduced KLF4 induction. Therefore, KLF4 expression seems to be regulated in a highly cell- and context-dependent manner. Overall, these data indicate that pneumococcal DNA induces KLF4 protein expression in a TLR9dependent manner in human lung epithelial cells.

Members of the Src protein kinase family have been suggested to participate in TLR9-related signalling $[18,19]$. In accordance with this suggestion, we observed that inhibition of Src by PP2 blocked KLF4 protein expression in pneumococci-infected cells. Thus, KLF4 induction in pneumococci-stimulated lung cells depended on a pneumococcus DNA-TLR9-MyD88-Src kinase pathway. TLR9 stimulation, however, does not appear to be solely anti-inflammatory, because intraperitoneal application of $\mathrm{CpG}$ oligodeoxynucleotide induces an inflammatory response in the lungs of mice [20]. Nevertheless, the observation that TLR9-deficient mice, but not knockout mice for TLR1, TLR2, TLR4 or TLR6, showed higher mortality in pneumococcal pneumonia points to an overall beneficial role of TLR9 in this disease [14].

Once induced, KLF4 was shown to bind to the il10 promoter, and KLF4 knockdown by siRNA blocked IL-10 expression in 

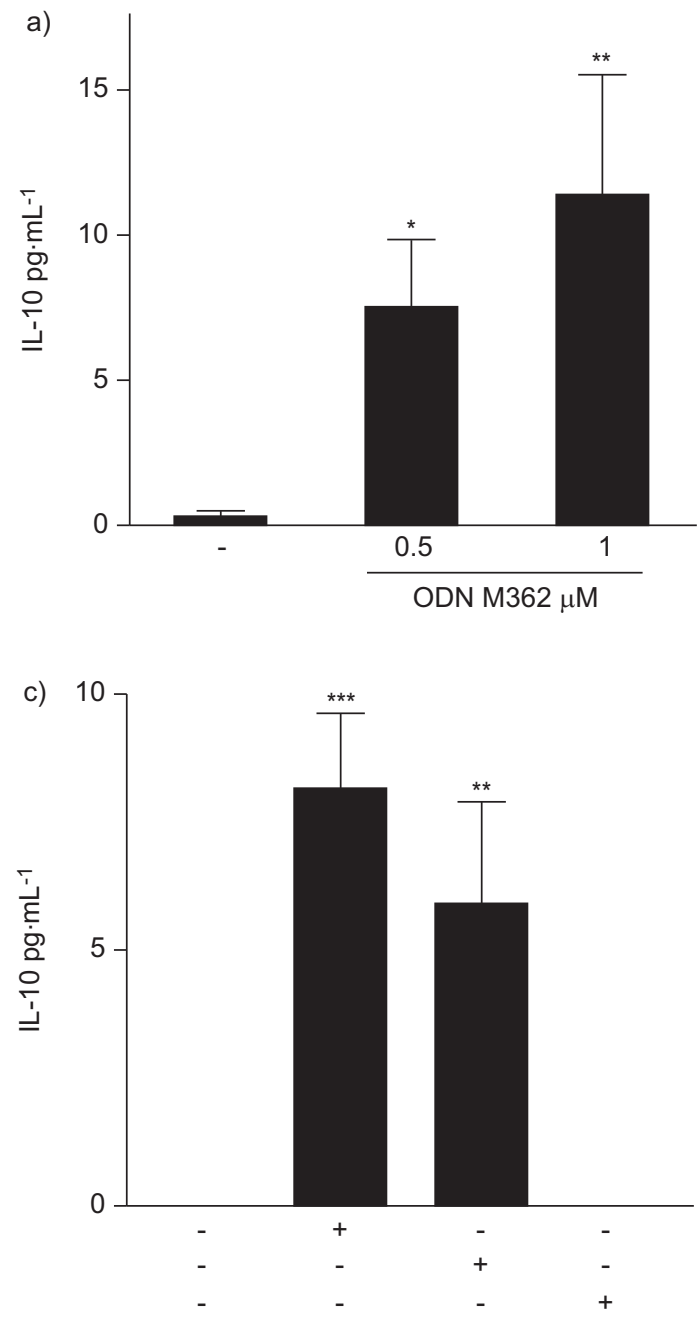
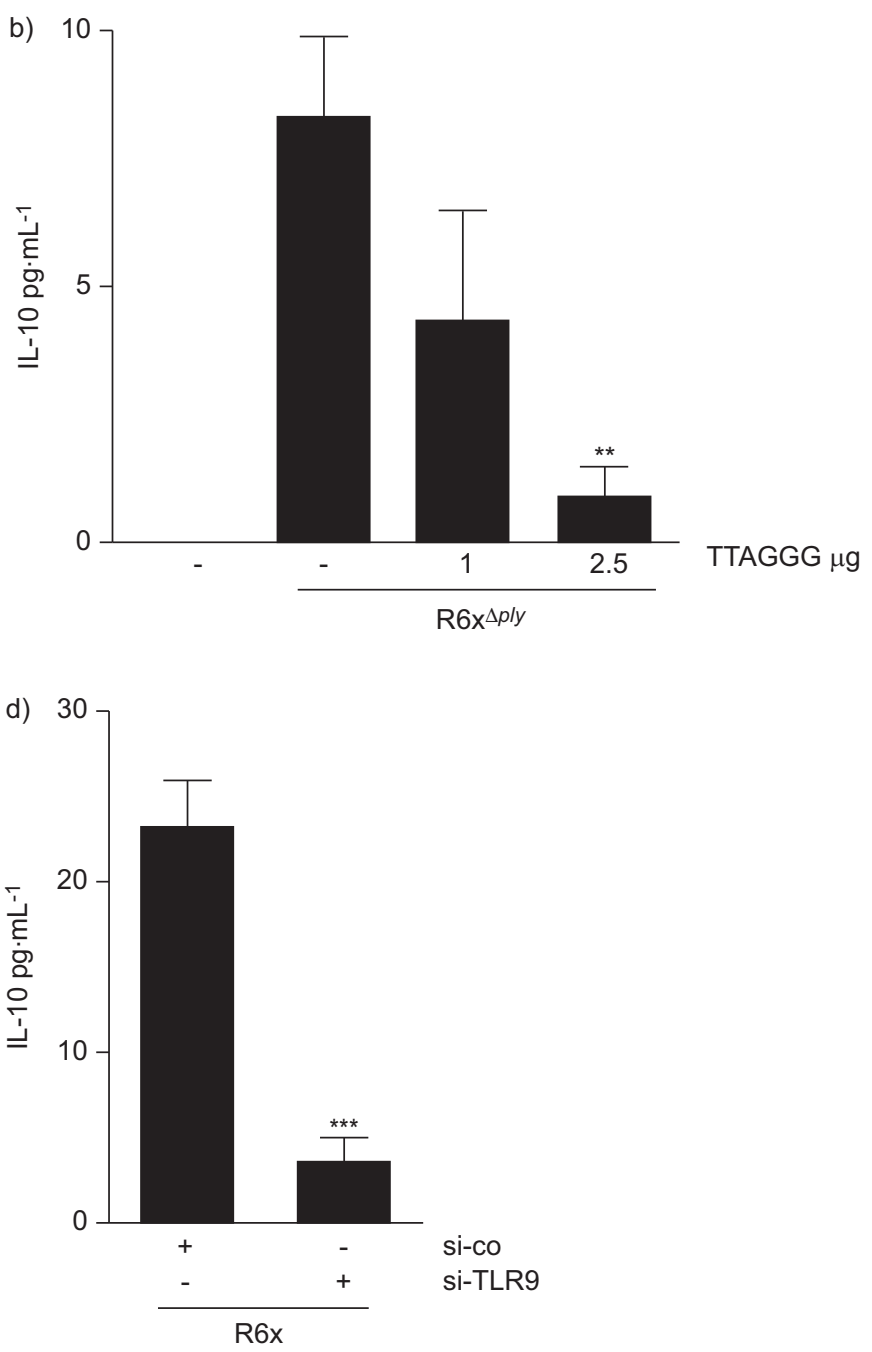

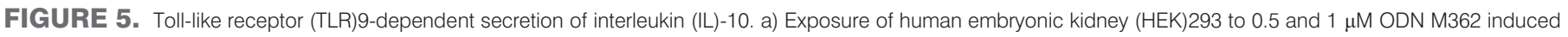

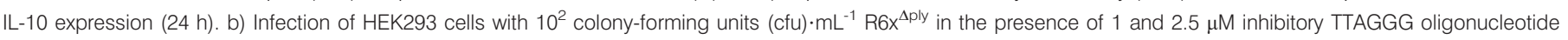

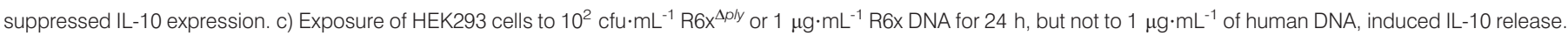

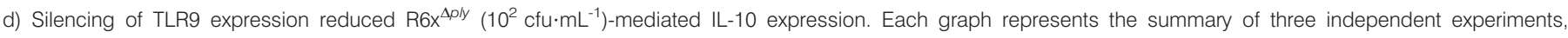
performed in duplicate. si-co: control siRNA; si-TLR9: small interferring TLR9; *: $p<0.05$; **: $p<0.01$; ***: $p<0.001$.

pneumococci-infected cells. In agreement with our data, LIU et al. [10] reported that KLF4 regulated the expression of IL-10 in macrophages. We observed increased expression of IL-10 in the BAL and lung tissue of pneumococci-infected mice and in infected human epithelial cells. Furthermore, CpG oligonucleotides and pneumococcal DNA induced IL-10 expression, whereas inhibitory TTAGGG oligonucleotide or TLR9- and MyD88 knockdown reduced IL-10 expression in our models. These observations are in line with MyD88 knockout cells producing less IL-10 following CpG DNA stimulation [21].

Finally, the inhibition of Src by PP2 also reduced pneumococcirelated IL-10 production. These observations confirmed the critical role of the TLR9-Src-KLF4 pathway for the induction of IL-10 (fig. 7). It maybe hypothesised that in vivo an IL-10 response is triggered by a preceding inflammatory reaction initiated by TLR activation, including TLR9. However, although an absence of TLR9 in mice reduced bacterial killing of pneumococci, it does not lead to increased cytokine production [14]. IL-10 is known to be increased in humans suffering from severe infections, including pneumonia, but its role is still discussed controversially. Early IL-10 application to humans exposed to LPS resulted in a reduced overall inflammatory response [22]. Experimental studies indicated that $i l-10$ reduces the antimicrobial capacity of phagocytotic cells and, therefore, high IL-10 levels in early phases of, for example, pneumonia may increase the risk of pathogen spread [23]. The observation that IL-10-1082 gene promoter polymorphism in humans (which is associated with early and high IL-10 levels) increases the risk of deleterious outcome in pneumonia furthermore indicates that early strong IL-10 release may be of disadvantage [24]. In severe pneumonia and pneumonia-related sepsis, both IL-6 and IL-10 levels were higher compared with uncomplicated pneumonia. One hypothesis is that, in these patients, increased IL-10 levels reflect a counterbalancing mechanism to avoid overwhelming inflammation. Thus, whereas extensive IL-10 expression may 
a)
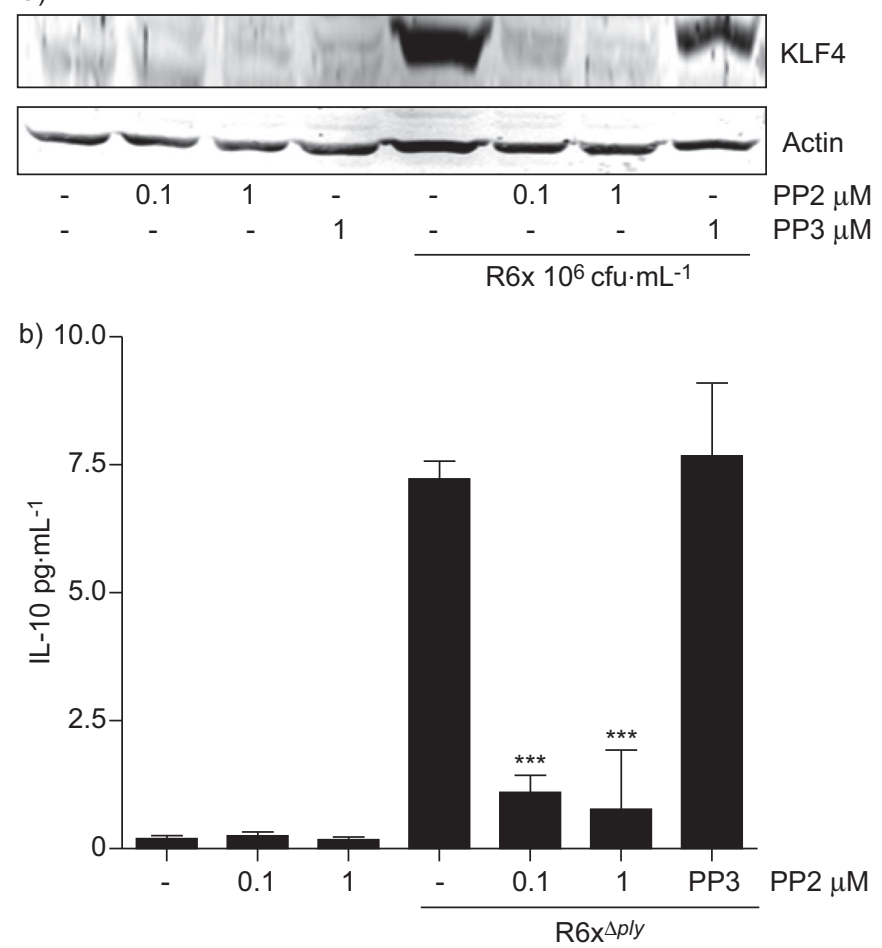

FIGURE 6. Src kinase inhibition reduced a) Krueppel-like factor (KLF)4 induction and b) interleukin (IL)-10 expression. Pre-incubation of bronchial epithelial cells (BEAS)-2B cells with $0.1-1 \mu \mathrm{M}$ Src kinase inhibitor PP2, but not $1 \mu \mathrm{M}$ of its inactive control PP3, for $1 \mathrm{~h}$ before infection reduced a) KLF4 expression and b) IL10 production in human embryonic kidney (HEK)293 cells. One representative blot out of three independent experiments is shown. Data represent mean \pm SEM of three independent experiments arranged in duplicate, and differences are indicated as follows: ${ }^{* *}: p<0.001$ compared with the specific effect of inhibitor.

reduce the host's capacity to respond appropriately to the pathogen in early phases of pneumonia, it may be important to reduce inflammation-related injury to the lung during the course of the disease. As the herein described KLF4-related IL10 response occurs with a delay, it could be viewed as a part of the counterbalancing IL-10 response in later phases.

In many organs, inflammation always bears the risk of causing deleterious tissue damage induced by the host response in addition to the damage caused by the pathogen itself. It would be of interest to analyse the role of KL4 in in vivo models of pneumococcal pneumonia. However, since conventional KLF4 knockout mice show a lethal phenotype [8], conditional KLF4 knockout mice may be used in these studies [25]. KLF4 seems to be involved in the immune regulation of immune cells [9, 10]. Moreover, for example, monocytotic cells are of great importance for lung tissue repair mechanisms [26, 27]. This raises the question about the role of epithelial versus myeloid KLF4 in pneumococcal pneumonia. Furthermore, it is important to follow up the role of KLF4 for apoptosis regulation in lung parenchyma cells, as well as myeloid-derived cells in pneumonia. By using cell/tissue-specific conditional KLF4 knockout models or, for example, reciprocal bone-marrow chimeric mice, these interesting questions will be addressed in further studies.

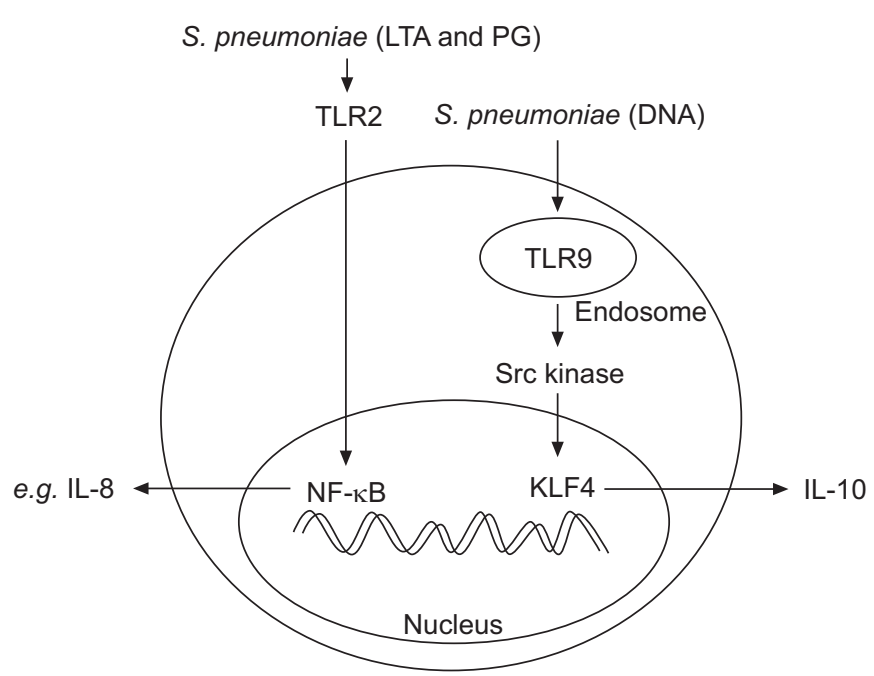

FIGURE 7. Pro- and anti-inflammatory signalling in lung epithelial cells. Stimulation of lung epithelial cells with Streptococcus pneumoniae leads to activation of different signalling pathways. Recognition of bacterial cell wall components like lipoteichoic acids (LTA) and peptidoglycan (PG) by TLR2 results in activation of the canonical nuclear factor (NF)- $\mathrm{kB}$ pathway and subsequent release of, for example, pro-inflammatory cytokines and chemokines, such as interleukin (IL)-8 [6]. A distinct pathway is triggered by recognition of bacterial DNA by TLR9, followed by activation of Src kinase and binding of KLF4 to the il-10 promoter, which finally leads to the release of the anti-inflammatory cytokine IL-10 (this study).

In conclusion, bacterial DNA-mediated TLR9-Src kinase activation induced KLF4 expression, thereby initiating expression of the central anti-inflammatory mediator IL-10. Thus, in addition to activating the innate immune response, atypical TLR-related signalling pathways may be important to control the inflammatory response in severe infections.

\section{SUPPORT STATEMENT}

This work was supported by the German Research Foundation (DFG) (SFB-TR84 to J. Zahlten, B. Schmeck, A.C. Hocke, M. Witzenrath, N. Suttorp and S. Hippenstiel), the Bundesministerium für Bildung und Forschung (FORSYS-Partner F-KZ 0315256, HMWK (LOEWEUGMLC) and DLZ to B. Schmeck; PROGRESS to N. Suttorp, B. Schmeck and S. Hippenstiel) and EU-Network CAREPNEUMO (to S. Hammerschmidt and S. Hippenstiel).

\section{STATEMENT OF INTEREST}

None declared.

\section{ACKNOWLEDGEMENTS}

We are grateful to J. Hellwig, A. Kühn, D. Stoll, F. Schreiber, K. Reppe and B. Gutbier (Dept of Internal Medicine/Infectious Diseases and Respiratory Medicine, Charité-Universitätsmedizin Berlin, Berlin, Germany) for excellent technical assistance. Parts of this work will be included in the MD thesis of R. Steinicke.

\section{REFERENCES}

1 Mathers CD, Loncar D. Projections of global mortality and burden of disease from 2002 to 2030. PLoS Med 2006; 3: e442.

2 Paterson GK, Mitchell TJ. Innate immunity and the pneumococcus. Microbiology 2006; 152: 285-293. 
3 Johansson N, Kalin M, Tiveljung-Lindell A, et al. Etiology of community-acquired pneumonia: increased microbiological yield with new diagnostic methods. Clin Infect Dis 2010; 50: 202-209.

4 Malley R, Henneke P, Morse SC, et al. Recognition of pneumolysin by Toll-like receptor 4 confers resistance to pneumococcal infection. Proc Natl Acad Sci USA 2003; 100: 1966-1971.

5 Mogensen TH, Paludan SR, Kilian M, et al. Live Streptococcus pneumoniae, Haemophilus influenzae, and Neisseria meningitidis activate the inflammatory response through Toll-like receptors 2 , 4, and 9 in species-specific patterns. J Leukoc Biol 2006; 80: 267-277.

6 Schmeck B, Huber S, Moog K, et al. Pneumococci induced TLRand Rac1-dependent NF- $\mathrm{kB}-$ recruitment to the IL-8 promoter in lung epithelial cells. Am J Physiol Lung Cell Mol Physiol 2006; 290: L730-L737.

7 Kaczynski J, Cook T, Urrutia R. Sp1- and Kruppel-like transcription factors. Genome Biol 2003; 4: 206.

8 Segre JA, Bauer C, Fuchs E. KLF4 is a transcription factor required for establishing the barrier function of the skin. Nat Genet 1999; 22 356-360.

9 Feinberg MW, Cao Z, Wara AK, et al. Kruppel-like factor 4 is a mediator of proinflammatory signaling in macrophages. $J$ Biol Chem 2005; 280: 38247-38258.

10 Liu J, Zhang H, Liu Y, et al. KLF4 regulates the expression of interleukin-10 in RAW264.7 macrophages. Biochem Biophys Res Commun 2007; 362: 575-581.

11 Zahlten J, Steinicke R, Opitz B, et al. TLR2- and nucleotide-binding oligomerization domain 2-dependent Kruppel-like factor 2 expression downregulates NF-kB-related gene expression. J Immunol 2010; 185: 597-604.

12 Witzenrath M, Schmeck B, Doehn JM, et al. Systemic use of the endolysin Cpl-1 rescues mice with fatal pneumococcal pneumonia. Crit Care Med 2009; 37: 642-649.

13 Santel A, Aleku M, Keil O, et al. A novel siRNA-lipoplex technology for RNA interference in the mouse vascular endothelium. Gene Ther 2006; 13: 1222-1234.

14 Albiger B, Dahlberg S, Sandgren A, et al. Toll-like receptor 9 acts at an early stage in host defence against pneumococcal infection. Cell Microbiol 2007; 9: 633-644.

15 Hornung V, Rothenfusser S, Britsch S, et al. Quantitative expression of Toll-like receptor 1-10 mRNA in cellular subsets of human peripheral blood mononuclear cells and sensitivity to $\mathrm{CPG}$ oligodeoxynucleotides. J Immunol 2002; 168: 4531-4537.

16 Taylor $\mathrm{T}, \mathrm{Kim} \mathrm{YJ}, \mathrm{Ou} \mathrm{X}$, et al. Toll like receptor 2 mediates proliferation, survival, NFkappaB translocation, and cytokine
mRNA expression in LIF-maintained mouse embryonic stem cells. Stem Cells Dev 2010; 19: 1333-1341.

17 McGettrick AF, O'Neill LA. Localisation and trafficking of Tolllike receptors: an important mode of regulation. Curr Opin Immunol 2010; 22: 20-27.

18 Chang YJ, Wu MS, Lin JT, et al. Induction of cyclooxygenase-2 overexpression in human gastric epithelial cells by Helicobacter pylori involves TLR2/TLR9 and c-Src-dependent nuclear factor- $\mathrm{\kappa}$ activation. Mol Pharmacol 2004; 66: 1465-1477.

19 Stovall SH, Yi AK, Meals EA, et al. Role of vav1- and src-related tyrosine kinases in macrophage activation by CpG DNA. J Biol Chem 2004; 279: 13809-13816.

20 Knuefermann P, Baumgarten G, Koch A, et al. CpG oligonucleotide activates Toll-like receptor 9 and causes lung inflammation in vivo. Respir Res 2007; 8: 72.

21 Lee KS, Scanga CA, Bachelder EM, et al. TLR2 synergizes with both TLR4 and TLR9 for induction of the MyD88-dependent splenic cytokine and chemokine response to Streptococcus pneumoniae. Cell Immunol 2007; 245: 103-110.

22 Pajkrt D, Manten A, van der Poll T, et al. Modulation of cytokine release and neutrophil function by granulocyte colony-stimulating factor during endotoxemia in humans. Blood 1997; 90: 1415-1424.

23 Wang E, Simard M, Ouellet N, et al. Modulation of cytokines and chemokines, limited pulmonary vascular bed permeability, and prevention of septicemia and death with ceftriaxone and interleukin-10 in pneumococcal pneumonia. J Infect Dis 2000; 182: $1255-1259$.

24 Schaaf BM, Boehmke F, Esnaashari H, et al. Pneumococcal septic shock is associated with the interleukin-10-1082 gene promoter polymorphism. Am J Respir Crit Care Med 2003; 168: 476-480.

25 Yoshida T, Kaestner KH, Owens GK. Conditional deletion of Kruppel-like factor 4 delays downregulation of smooth muscle cell differentiation markers but accelerates neointimal formation following vascular injury. Circ Res 2008; 102: 1548-1557.

26 Cakarova L, Marsh LM, Wilhelm J, et al. Macrophage tumor necrosis factor- $\alpha$ induces epithelial expression of granulocytemacrophage colony-stimulating factor impact on alveolar epithelial repair. Am J Respir Crit Care Med 2009; 180: 521-532.

27 Marsh LM, Cakarova L, Kwapiszewska G, et al. Surface expression of CD74 by type II alveolar epithelial cells: a potential mechanism for macrophage migration inhibitory factor-induced epithelial repair. Am J Physiol Lung Cell Molec Physiol 2009; 296: L442-L452. 